\title{
Quantum Boson Data Aggregation Scheduling in UOSN
}

\author{
. ${ }^{1}$ S. Madhavi \\ 1Department of Computer Science and Engineering , P . V . P . Siddhartha \\ Institute of Technology, Andhra Pradesh, India,
}

\begin{abstract}
Data Aggregation Scheduling in Ubiquitous sensor networks is a major research interest for many researchers with the objective of reducing energy consumption and maximizing the network lifetime. Also the capacity of a channel for transmitting quantum states called the quantum capacity should be maximized,The sensor nodes should periodically forward data to the base station. Since battery power and bandwidth are the two resources to be effectively utilized the sensor nodes forwards data to the nearest neighbor node which inturn forwards the data to its neighbors and this repeats until all the sensors data aggregates at the base station. In a large network scheduling the sensor nodes for forwarding the data is very much essential and since the topology of the USN changes dynamically, finding such a schedule is also very difficult. In [1] the authors proposed a bosonic network model for communication. In this paper we present a quantum bosonic data aggregation method for constructing the optimal schedule of nodes for data aggregation called QBDAS (Quantum Boson Data aggregation scheduling) protocol. The main idea is to search a optimal schedule through utilizing the observable or measurable physical properties the network topology.
\end{abstract}

Keywords: USN, $B C, Q B D A S, Q K D, A P S P$

\section{Introduction}

The two important objectives in ubiquitous sensor networks(USN) are to maintain network reachability and optimizing the network lifetime. Earlier several centralized and distributed scheduling algorithms are proposed for data aggregation in USN $[2,3,5,6,8$, $9,12,20-24]$. In $[4,33]$ we presented a novel distributed data aggregation scheduling protocol using a power control collision-interference-free model, which is adaptive to the rate and power control. Since the ubiquitous nodes changes its state dynamically, finding a solution to the above mentioned tasks is very difficult. There exist many mathematical algorithms for the shortest path problem such as Dijkstra , Bellman-Ford, FloydWarshalls and APSP (All pair shortest path) problem which runs in $\mathrm{O}(\mathrm{MN}+\mathrm{N} 2 \log \log$ $\mathrm{N})$ time, where $\mathrm{M}$ and $\mathrm{N}$ are the numbers of edges and nodes of the graph, respectively. The Chan's algorithm has the time complexity in $\mathrm{O}(\mathrm{N} 3 / \log 2 \mathrm{~N})$ and the algorithm by Seidel has $\mathrm{O}^{\sim}\left(\mathrm{N}\right.$ !) time complexity. Shoshan and Zwick proposed an $\mathrm{O}^{\sim}(\mathrm{WN}$ !) algorithm. In this paper, we assumed an itinerant bosonic network model and designed a protocol for obtaining an optimal schedule for data aggregation problem. An optimization function is used to find the optimal schedule step by step.

The idea of a quantum computer was first proposed by Richard Feynman in 1981 [7], which can accurately simulate quantum mechanical systems that can't be possible on a classical computer. QKD (quantum key distribution) systems use quantum bits for secure quantum communications using freespace and fiber optic links.

Several quantum algorithms have emerged during these past few years for establishing system for secure quantum communications. An intermediate quantum computer called a "boson sampling" machine is developed which stands between classic computers and quantum computers[11]. The device consists of a network of beam splitters that converts one set of photons arriving at a number of parallel input ports into a second set leaving via 
a number of parallel outputs[11]. Its task is to work out the probability that a certain input configuration will lead to a certain output[11].

Centrality measures[10] are used to decide which is the best node in the network. Some of the measures of centrality are

- Degree centrality. Degree centrality of a node in a network is the number of links (vertices) incident on the node.

- Closeness centrality. Closeness centrality determines how "close" a node is to other nodes in a network by measuring the sum of the shortest distances (geodesic paths) between that node and all other nodes in the network.

- Between's[10,16]. Between's centrality determines the relative importance of a node by measuring the amount of traffic flowing through that node to other nodes in the network. This is done by measuring the fraction of paths connecting all pairs of nodes and containing the node of interest. Group Between's centrality measures the amount of traffic flowing through a group of nodes. Between's Centrality (BC) stands for the ability of an individual node to control the communication flow in the networks. Formally, for a node $\mathrm{v}$ it denoted the total portion of shortest-paths between every pair of nodes in the network that pass through v. Holme [14] have shown that Betweenness is highly correlated with congestion in particle hopping systems.

Let $\sigma_{i, j}$ be the number of shortest paths between the origin vertex $i$ and the destination vertex $\mathrm{j}$ where $\mathrm{i}, \mathrm{j} \in \mathrm{V}$.

Let $\sigma_{i, j}(v)$ be the number of routes from $i$ to $j$ that pass through the vertex $v$.

The Betweenness centrality[10][16]can hence be expressed by the following equation:

$$
B C(v)=\sum_{i, j \in V} \frac{\sigma_{i, j}(v)}{\sigma_{i, j}} \quad \rightarrow 1
$$

- Eigenvector centrality. Eigenvector centrality is a more sophisticated version of degree centrality where the centrality of a node not only depends on the number of links incident on the node but also the quality of those links. This quality factor is determined by the eigenvectors of the adjacency matrix of the network.

- Katz centrality. Katz centrality of a node is measured by summing the geodesic paths between that node and all (reachable) nodes in the network. These paths are weighted, paths connecting the node with its immediate neighbors carry higher weights than those which connect with nodes farther away from the immediate neighbors.

The Physical distance metric should be replaced by the path length for better results. A path is a route that runs along the links of a network. For an undirected and unweighted network, the path length is defined as the number of edges in a path connecting any two vertices $i$ and $j$ is called the path length and in weighted graphs, it is defined as the sum of all the weights on the edges to get the path lengths. The average of the shortest path length between two nodes over the entire network is called the characteristic path length[10][16]. It can be used as a measure of efficiency of the network. Hence the path length $\mathrm{D}$ is the average of the shortest path lengths in a network $[10,16]$, defined as

$$
D=\frac{2}{N(N-1)} \sum_{i<j} d_{i, j} \rightarrow 2
$$

where matrix $\mathrm{d}_{\mathrm{ij}}$ is obtained from Dijkstra's algorithm.

In this paper our optimization function chooses the optimal node measuring the intensity of the light passing through that node. The rest of the paper is organized as follows 
In section 2 we discussed about the proposed quantum bosonic network model . In section 3 we discussed about the proposed optimized function to find the schedule for data aggregation problem in USN. In section 4 we discussed about the conclusion.

\section{Proposed Ubiquitous Quantum Bosonic Optical Communication Sensor Network Model}

Consider a ubiquitous quantum bosonic [17-19] optical communication sensor network with $n$ arbitrarily distributed ubiquitous nodes[1]. Let a graph $\mathrm{G}=(\mathrm{V}, \mathrm{E})$ denote an optical network where edges $E=\left\{e_{0}, e_{1}, e_{n-1}\right\}$ represent optical fibers where $e_{i}$ denotes the edge between two nodes and nodes $\mathrm{V}=\left\{\mathrm{v}_{0}, \mathrm{v}_{1}, \mathrm{v}_{\mathrm{n}-1}\right\}$ represent optical units where $\mathrm{V}_{\mathrm{i}}$ denotes node i. In this work we have selected photons called as free boson as the physical entity. Our cluster head searching method realize on measuring the intensity of light waves on relevant nodes. The cluster heads are obtained by measuring the intensity of the photons. The proposed network model in this paper is based on an Anderson-like itinerant bosonic system in which an optimal schedule is selected using a boson's node optimization function.

Similarly

The degree of a node is defined as the number of nodes to which it is connected.

The network adjacency matrix $\mathrm{A}$ is defined as

- The matrix element $A_{i j}$ is set to unity if $i=j$.

- $\quad$ Otherwise it is set to 0 . by

Since the network is unidirected, $\mathrm{A}^{\mathrm{T}}=\mathrm{A}$ holds true. The degree for a node $\mathrm{i}$ is given

$$
\mathrm{K}_{\mathrm{i}}=\sum_{i \neq j} A_{i j}
$$

Bosons[13] are identical particles which can share their quantum states. The BoseEinstein Statistics describes the behaviour of the bosons. The statistical properties are described as Bose-Einstein statistics and the Hamiltonian for such a system is

$$
H=\sum_{i j v} b_{i, j}^{v} c_{i v}^{\perp} c_{j v}+\frac{1}{2} \sum_{i \mu v} S_{\mu v} \eta_{i p}\left(\eta_{i v}-\delta_{\mu v}\right)=H_{0}+H_{\mathrm{int}} \quad \rightarrow 4
$$

Where $b^{v}{ }_{i j}$ are the hopping amplitudes[1] of the bosons and $\mathrm{S}$ denotes the local density interactions between the $\mu$ and $\mathrm{v}$ - bosons. For natural basis of a single particle states , the hopping amplitudes and Hamiltonian in matrix[1] form are defined as follows

$$
\begin{aligned}
t_{i j}=-A_{i j} \text { if } \mathrm{i} \neq \mathrm{j} & \\
\mathrm{t}_{\mathrm{ij}}=\frac{\sum_{l \neq i} A_{i l} \sqrt{K_{l}}}{\sqrt{K_{i}}} \text { if } \mathrm{i}=\mathrm{j} & \rightarrow 5
\end{aligned}
$$

In the above equation larger value of the numerator suggests that the particle hopping randomly has less chance to return to the node and the visibility of the particle at this node is decreased.

The Hamiltonian for the single particle state is written as follows 


$$
H=\left(\begin{array}{ccccc}
\frac{\sum_{l}^{i} A_{i l} \sqrt{K_{l}}}{\sqrt{K_{l}}} & -A_{12} & \ldots & \ldots & \ldots \\
-A_{21} & \frac{\sum A_{21} \sqrt{K_{l l}}}{\sqrt{K_{2}}} & \ldots & \ldots & \ldots \\
\ldots & \ldots & & \ldots & \ldots \\
\ldots & \ldots & \ldots & \ldots & \ldots \\
-A_{N 1} & -A_{N 2} & \ldots & \ldots & \frac{\sum_{l}^{i} A_{N l} \sqrt{K_{l}}}{\sqrt{K_{N}}}
\end{array}\right) \rightarrow 6
$$

The spectral decomposition of the one particle density matrix[1] is defined as follows

The $\mathrm{DS}_{\mathrm{ij}}-\sum_{l} \lambda_{l} \theta_{i l}^{*} \theta_{j l}$ where $\propto$ denotes the wave function.

For single particle systems $\mathrm{H}$ can be written as

$$
H=-b \sum_{i j} c_{i}^{\perp} c_{j} \quad \rightarrow 7
$$

And the energy [21] can be written as

$$
\mathrm{E}=-b \sum_{i, j} D S_{i j} \quad \rightarrow 8
$$

Hence the following optimization function is used to find the node which has high light intensity[1]

$$
\operatorname{HLIN}(\mathrm{dst}, \Delta \mathrm{t}, \mathrm{src}, 0)=\quad \sum_{n}\left(<f \mid \theta_{n}>\right)<\theta_{n} \mid S>e^{i\left(E_{n}-E_{0}\right) \Delta t} \quad \rightarrow 9
$$

Where

$$
\begin{array}{ll}
\theta_{0}=\frac{1}{\sqrt{\sum_{n} \sqrt{K_{n}}}} \sum_{i}^{N} \sqrt{K_{i} \mid i} & \rightarrow 10 \\
\mathrm{~K}_{\mathrm{i}}=\sum_{i \neq j} w_{i j} A_{i j} & \rightarrow 11
\end{array}
$$

A shortest path $\mathrm{P}$ between node $\mathrm{v} 1$ and $\mathrm{vk}$ is obtained as follows

such that

$$
\left(\mathrm{v}_{1},\left(\mathrm{v}_{1}, \mathrm{v}_{2}\right), \mathrm{v}_{2}, \ldots,\left(\mathrm{v}_{\mathrm{n}-1}, \mathrm{v}_{\mathrm{n}}\right), \mathrm{v}_{\mathrm{n}}\right)
$$

$$
\left(v_{i}, v_{i+1}\right) € E \quad \text { for } i=1, \ldots, n-1 .
$$

The path is from the nodes $\mathrm{i}$ to $\mathrm{j}$ where $1<=\mathrm{i}<=\mathrm{j}<=\mathrm{n}$.

Nodes $i$ and $j$ are called the origin and terminal nodes of the path $p$ resp.

A path from $\mathrm{i}$ to $\mathrm{j}$ is denoted by $\mathrm{i}-\mathrm{j}$ path and the distance from $\mathrm{i}$ to $\mathrm{j}$ is denoted by $\mathrm{d}(\mathrm{i}, \mathrm{j})$, $\mathrm{N}(\mathrm{v})=\left\{\mathrm{w} \mid \mathrm{A}_{\mathrm{wv}}, \mathrm{w}\right.$ not eqaul to $\left.\mathrm{v}\right\}$ is the set of neighbour nodes of $\mathrm{v}$ 
An optimal schedule is a schedule with[22][23][24][25][26]

a.Minimum number of timeslots(frame length)

b. Maximum number of concurrent transmissions per each timeslot.

\section{Distributed Algorithm.}

In this work, we build an optimal ubiquitous quantum bosonic optical communication sensor network. The sensor nodes aggregates data to the base station. Generally the communication network is divided into clusters and each cluster has a cluster head.

Given a UQBOSN tree then the procedure Optimal_SHDL(ConCHeadTree) consists of the following phases

1. Head Selection Phase: Construct one or more ConCHeadTrees and from each ConCHeadTree identify one or more ConCHeadnodes. A node which has high light intensity through it is called the Head node and it is obtained by the method called Cluster Head selection algorithm. All such head nodes are identified in the communication network. The region around such Head nodes are treated as one cluster. This cluster head is called as Head Node in this paper. There are many existing algorithms for obtaining optimal number of clusters in a network. If $\mathrm{H}$ denotes the maximum hop length in a cluster and if $\mathrm{L}$ denotes the maximum hop length in the communication network then each cluster may satisfy a property like $3<=\mathrm{H}<=\mathrm{L} / 4$ to specify the cluster boundary.

A node cannot belong to two or more clusters. If it has paths to two or more clusters then it belongs to one cluster to which it is geographically nearest. We also used an optimization function to find out the node that has the optimum light intensity. The following is the procedure to select a Head node from the network. This algorithm is a iterative procedure and ends when the total network is grouped into clusters and every node belongs to any one cluster.

The Cluster Head Selection algorithm is as follows

Step 1. For src in $V_{i}, i=1$ to $n$ do the following steps through step 7.

Step 2. Let src denote a source node and dst denote a destination node from the set of vertices $\mathrm{V}$ in a given network.

Step 3. Let Neigh(src) $=$ FindNeighbours(src) for all $i=1,2,3, \ldots \mathrm{N}$ where $\mathrm{N}$ denotes the total number of nodes in the network.

Step 4. Call Intensity (dst, $\Delta \mathrm{t}, \mathrm{src}, 0)$

Step 5. Find the intensity of light between dst and any neighbor nodes Neigh(src) $)_{i}$ where $\operatorname{Neigh}(\mathrm{src})_{i}$ belongs to $\mathrm{Neigh}(\mathrm{Src})_{i}$ and $\mathrm{i}=1,2,3,4$, ---- Ksrc where Ksrc is the degree of the node src

Step 6. Use the optimization function HLIN(dst, $\Delta \mathrm{t}, \mathrm{src}, 0)$ to find the neighbour that has the optimal intensity of light. Denote the neighbor as ConCHeadnode and then set src to ConCHeadnode

Step 7. Goto step 2 if none of the neighbours of src is dst else output the sequence of ConCHeadnode

Step 8. End.

Aggregation Scheduling Phase: Construct a schedule[4] with the list of active nodes for each ConCHeadnode. Once after identifying the clusters and head nodes called ConCHeadnode, all the nodes aggregates data to the head nodes. The concHeadnode 
acts as the cluster head. Using the scheduling method spiecified in [4] all the nodes are scheduled inorder to see that two or more nodes will not interfeare while transmitting.

Each schedule specifies

-When cluster head node should transmit,

- How the cluster head node gathers data from its neighbors.

An optimal network ensures optimal transmission ranges leading to energy savings of a node and the full reachability of the network. In UQOCSN such a set of schedules are constructed and from which an optimal schedule is the one maximizes the performance of the network. Our distributed algorithm is an iterative process and ends when the complexity of the ConCHeadTree cannot be further decreased. Much research is carried for finding data aggregation schedules like in [25][26][27][28][29][30][31][32]. Due to various noisy and lossy quantum channels communication with quantum information is very difficult. Hence development of the protocols for such quantum communcaiton is very much essential.

An optimal schedule is constructed with edges from $\mathrm{E}$. The total transmission time $\mathrm{T}$ consists of slots $\left\{\mathrm{t}_{0}, \ldots \mathrm{t}_{\mathrm{T}-1}\right\}$ and the total schedule $\mathrm{S}=\left\{\mathrm{S}_{0}, \mathrm{~S}_{1}, \ldots, \mathrm{S}_{\mathrm{T}-1}\right\}$, where $\mathrm{S}_{\mathrm{t}}$ denotes the subset of nodes in $\mathrm{V}$ scheduled to transmit in time slot $\mathrm{t}_{\mathrm{i}}$.

The optimal schedule satisfies the following conditions.

-Each active node may be scheduled more than once but must be scheduled at least once

- A node cannot act as a transmitter and a receiver in the same time slot, in order to avoid the primary interference [4].

A Head node vi transmits to the Base station only after all its neighbors have been scheduled [4]. An optimal schedule always improves the network capacity and also best utilizes the hardware resources at each ubiquitous node. The proposed method prolongs network lifetime by implementing energy consumption. Generally time is slotted to intervals where $\mathrm{L}$ denotes the length of the interval.

All noninterfearing nodes ui are scheduled to send their aggregated data to any of their neighbors, vi. There exist one or more ConcHeadnodes to forward the aggregated data to the base station and each ConCHeadnode have zero, one or more neighbors from where it gathers the data. Generally a ConCHeadnode is selected based on a criterion that improves the utilization of the hardware resources and maximizes the network throughput.

Let AVij , for $i, j=1 . . n$ denote the set of active nodes for each ConCHeadnodei. Assign a timeslot in the schedule for each AVij. These slots are allocated in such a way that there are no interferences among the concurrent transmitters. The schedule consists of one or more slots. And at the end of the two phases a set of schedules are identified.

\section{Procesure Intensity $\left(V_{i}\right.$ a destination node $, \Delta t, V_{i}$ a source node. 0$)$}

Step 1. Calculate the value of $t_{i j}$ as given in equation 5 for a given source node $V_{i}$ and destination node $\mathrm{V}_{\mathrm{j}}$.

Step 2. Calculate the value of $E$ as given in equation 8 .

In the process of aggregating data to the base station a Node $V_{i}$ chooses one of its neighbours $\mathrm{N}_{\mathrm{l}}$ or $\mathrm{N}_{\mathrm{k}}$ such that at any instant where

If $E_{i j}(k-1)<\mathrm{E}_{i l}(k-1)+E_{l j}(k-1)$

Then,

$$
E_{i j}(k)=E_{i j}(k-1)
$$


Otherwise,

$$
E_{i j}(k)=E_{i l}(k-1)+E_{l j}(k-1)
$$

Step 3. When all the nodes in the path have been covered, the index of each edge gives the maximum intensity of light for the paths from node $i$.

Step 4. Repeat steps 1 to 3 with different nodes until all the nodes are covered.

Step 5. The sum of indices for all possible iterations gives the complete intensity of light value between each pair of nodes.

Step 6. End.

\section{Conclusion}

We discussed the problem data aggregation in an ubiquitous quantum bosonic optical communication sensor network with $\mathrm{n}$ arbitrarily distributed ubiquitous nodes. Our aggregation problem is based on measuring the intensity of light waves on relevant nodes. The cluster heads are obtained by measuring the intensity of the photons. All the nodes aggregated data to the cluster head which inturn aggregates data to their cluster heads and finally to the base station. An optimal schedule for such aggregation is obtained using a boson's node optimization function. The main idea is to search a optimal schedule through utilizing the observable or measurable physical properties the network topology. This method not only provides a novel method for the data aggregation problem using quantum mechanics in quantum optical communication sensor networks.

\section{References}

[1] Jiang X, Wang H L, Tang S T, Zhang Z L, Ma L L, Tian G S and Zheng Z M, "Detecting the structure of complex networks by quantum bosonic dynamics", Physica A, vol. 389, (2010), pp. 2465-71.

[2] S. C.-H. Huang, P.-J. Wan, C. T. Vu, Y. S. Li and F. Yao, "Nearly Constant Approximation for Data Aggregation Scheduling in Wireless Sensor Networks," Proceedings of 26th IEEE International Conference on Computer Communications, (2007); Anchorage.

[3] H.-. Lee, J.-H. Hwang, H. Yoe,"Energy Efficient MAC Protocol for Ubiquitous Agriculture", International Journal of Smart Home, vol.4, no.3, (2010).

[4] S.Madhavi, "Dynamic Distributed Power Adaption for Data Aggregation in Ubiquitous Sensor Networks", Asia-pacific Journal of Multimedia Services Convergent with Art, Humanities, and Sociology, vol.3, no.2, (2013), pp. 47-60.

[5] M. Ren, L. Guo, J. Li ,'A New Scheduling Algorithm for Reducing Data Aggregation Latency in Wireless Sensor Networks", Int. J. Communications, Network and System Sciences, vol. 3, (2010), pp. 679-688.

[6] S. Kumar, S. Chauhan, "A Survey on Scheduling Algorithms for Wireless Sensor Networks", International Journal of Computer Applications, vol. 20, no. 5, (2011).

[7] M. Oskin, "Quantum Computing - Lecture Notes", University of Washington.

[8] R. Sheikhpour, S. Jabbehdari and A. K. Zadeh,"A Cluster-Chain based Routing Protocol for Balancing Energy Consumption in Wireless Sensor Networks", International Journal of Multimedia and Ubiquitous Engineering, vol. 7, no. 2, (2012).

[9] A. K. Tripathy and S. Chinara, "Comparison of Residual Energy-Based Clustering Algorithms for Wireless Sensor Network", ISRN Sensor Networks Volume, (2012).

[10] Y. Altshuler, R. Puzis, Y. Elovici, S. Bekhor, and Alex, "Augmented Betweenness Centrality for Mobility Prediction in Transportation Networks", Finding Patterns of Human Behaviors in Network and Mobility Data (NEMO)", (2011),

[11] "Boson sampling offers shortcut to quantum computing", PhysicsWorld.com, (2013).

[12] T. Kim, H. Lim, J. C. Hou, "Improving Spatial Reuse through Tuning Transmit Power, Carrier Sense Threshold and Data Rate in Multihop Wireless Networks", MobiCom'06, (2006); Los Angeles, California , USA.

[13] A. Halu, L. Ferretti, A. Vezzani and G. Bianconi, "Phase diagram of the Bose-Hubbard model on complex networks", Europhysics Letters, vol. 99, (2012), pp. 18001.

[14] P. Holme, "Congestion and centrality in traffic flow on complex networks", Advances in Complex Systems, vol. 6, no. 2, (2003), pp. 163-176. 
[15] U. Brandes, "On variants of shortest-path betweenness centrality and their generic computation", Social Networks, vol. 30, no. 2, (2008), pp.136-145.

[16] U. Brandes, "A Faster Algorithm for Betweenness Centrality", Journal of Mathematical Sociology, vol. 25, no. 2, pp. 163--177, (2001).

[17] Y. Yu, B. Krishnamachari and V. K. Prasanna, "En-ergy-Latency Trade-Offs for Data Gathering in Wireless Sensor Networks", Proceedings of 23rd Annual Joint Conference of the IEEE Computer and Communications Societies, (2004); Hong Kong.

[18] X.-G. Wen, "Quantum Field Theory of Many-Body Systems", (2007); Oxford University, New York.

[19] C. Nayak, S. H. Simon and A. Stern, M. Freedman, and S. Das Sarma, "Non-Abelian anyons and topological quantum computation", Rev. Mod. Phys., vol. 80, (2008), pp. 1083.

[20] J. Alicea, "New directions in the pursuit of Majorana fermions in solid state systems", Rep. Prog. Phys. 75, (2012).

[21] X. Yu, S. Mehrotra, and N. Venkatasubramanian, "Sensor scheduling for aggregate monitoring in wireless sensor networks", Proc. of the 19th Int. Conf. on Scientific and Statistical Database Management (SSDBM'07), (2007).

[22] D. J. Watts and S. H. Strogatz, "Collective dynamics of "small-world" networks Nature (London), vol. 393 , no. 440, (1998).

[23] X. Chen, X. Hu and J. Zhu, "Minimum data aggregation time problem in wireless sensor networks," Lecture Notes in Computer Sciences, vol. 3794, (2005), pp. 133-142.

[24] S. C.-H. Huang, P.-J. Wan, C. T. Vu, Y. Li, and F. Yao, "Nearly constant approximation for data aggregation scheduling in wireless sensor networks", Proc. of the 26th Conf. on Computer Communications (INFOCOM'07), (2007).

[25] B. Yu, J. Li, and Y. Li, "Distributed data aggregation scheduling in wireless sensor networks", Proc. of the 28th Conf. on Computer Communications (INFOCOM'09), (2009).

[26] Y.-T. Chen , M.-F. Horng, C.-C. Lo, S.-C. Chu , J.-S. Pan and B.-Y. Liao, "A Transmission Power Optimization with a Minimum Node Degree for Energy-Efficient Wireless Sensor Networks with FullReachability".

[27] P.-J. Wan, S. C.-H. Huang, L. Wang, Z. Wan, and X. Jia, "Minimum latency aggregation scheduling in multihop wireless networks," Proc. of the 10th ACM Int. Symposium on Mobile Ad Hoc Networking and Computing (MOBIHOC'09), (2009).

[28] R.W. Floyd, "Algorithm 97: Shortest path", Communications of the ACM, vol. 5, no. 6, (1962), p. 345.

[29] L. C. Freeman, "A Set of Measures of Centrality based on Betweenness," Sociometry, vol. 40, no. 1, (1977), pp. 35-41.

[30] A. M. Berman, "Lower and upper bounds for incremental algorithms", Computer Science, The State University of New Jersey at Rutgers, New Brunswick, NJ, PhD Dissertation, (1992).

[31] G. Ramalingam and T. Reps, "On the Computational Complexity of Incremental Algorithms", CS, Univ. of Wisconsin at Madison, Tech. Report, (1991).

[32] P. Fraigniaud, C. Gavoille, A. Kosowski, E. Lebhar and Z. Lotker, Universal augmentation schemes for network navigability: overcoming the n-barrier 19th Annu. ACM Symp. on Parallelism in Algorithms and Architectures, (2007); San Diego, CA.

[33] Z. Chen, G. Yang, L. Chen, and J. Wang, "An Algorithm for Data Aggregation Scheduling with Longlifetime and Low-latency in Wireless Sensor Networks", International Journal of Future Generation Communication and Networking, vol. 5, no. 4, (2012).

[34] S. Madhavi, "Secured Data Aggregation Scheduling in Ubiquitous Quantum Sensor Networks", Asiapacific Journal of Multimedia Services Convergent with Art, Humanities, and Sociology, vol.4, no.1, (2014), pp. 17-30.

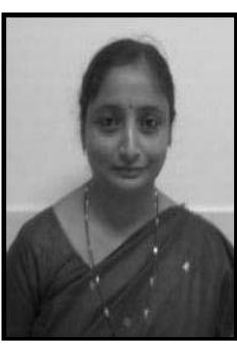

S.Madhavi, she received her M.S.degrees from the BITS, Pilani in 1999 and $\mathrm{PhD}$ in Computer Science and Engineering in 2011. She is presently working as a Professor in the department of Computer Science and Engineering, P.V.P. Siddhartha Institute of Technology, India. She published 19 papers and one edited book. Her research interests are Wireless Sensor Networks, Quantum Cryptography, Neural Networks and Fuzzy logic and Object Oriented Analysis and Design Patterns. 